\title{
ОПРЕДЕЛЕНИЕ ВОЗМУЩАЮЩЕЙ НАГРУЗКИ, НЕОБХОДИМОЙ ДЛЯ УПЛОТНЕНИЯ ПОЛИМЕРНОГО БЕТОНА
}

\section{А. Г. Маслов, Д. В. Савелов}

Кременчугский национальный университет имени Михаила Остроградского

ул. Первомайская, 20, г. Кременчуг, 39600, Украина. E-mail: dvsavelov@gmail.com

На основании детального анализа существующих реологических моделей уплотняемых сред предложена реологическая модель вибрируемой полимерной бетонной смеси, которая наряду с обратимой и необратимой деформацией учитывает обратимую высокоэластичную деформацию полимерного вяжущего под нагрузкой. Для предложенной реологической модели полимерной бетонной смеси, которая учитывает её упругие, вязкие и пластические свойства, составлено волновое уравнение колебаний, для теоретического анализа которого применен метод решения в комплексных функциях. В результате проведенных исследований найдено решение волнового уравнения колебаний и выведены аналитические выражения для определения амплитудных напряжений, которые возникают на поверхности и в основании уплотняемого слоя полимерного бетона в зависимости от координаты. На основании условия уплотняемости получено аналитическое выражение для определения амплитуды возмущения поверхности полимерного бетона, которая необходима для его полного уплотнения. По найденому значению амплитуды возмущения определяется амплитудное значение возмущающей силы вибраторов вибрационного рабочего органа.

Ключевые слова: реологическая модель, полимерный бетон, напряжение, деформация, амплитуда возмущения.

\section{ВИЗНАЧЕННЯ ЗБУДЖУЮЧОГО НАВАНТАЖЕННЯ, НЕОБХІДНОГО ДЛЯ УЩІЛЬНЕННЯ ПОЛІМЕРНОГО БЕТОНУ}

\section{О. Г. Маслов, Д. В. Савєлов}

Кременчуцький національний університет імені Михайла Остроградського

вул. Першотравнева, 20, м. Кременчук, 39600, Україна. E-mail: dvsavelov@gmail.com

На підставі ретельного аналізу науково-технічної літератури встановлено, що на сьогоднішній день для виробництва виробів з полімерного бетону застосовують технологію вібраційного ущільнення цементного бетону або залізобетону, яка не враховує специфічні особливості та властивості полімерної бетонної суміші та не дозволяє отримувати з неї якісні та надійні полімерні бетонні вироби. На підставі аналізу поведінки матеріалів та існуючих реологічних моделей ущільнюваних середовищ визначено, що для полімерної бетонної суміші, що вібрує, запропонована реологічна модель Зінера, у якій під дією зовнішнього навантаження разом з оборотною та необоротною деформацією враховується оборотна високо еластична деформація полімерного в'яжучого. Встановлено, що у існуючих дослідженнях реологічна модель Зінера не застосовувалась для опису поведінки полімерної бетонної суміші, як суцільного середовища, а іiі коливання, під впливом вібрації, не описувались хвильовим рівнянням коливань. Для запропонованої реологічної моделі ущільнювальної полімерної бетонної суміші, яка враховує іiї пружні, в'язкі та пластичні властивості, складено хвильове рівняння коливань, для теоретичного аналізу якого застосований метод розв'язання у комплексних функціях. У результаті проведених теоретичних досліджень визначено фазову швидкість поширення збудження та коефіцієнт поглинання, який характеризує зменшення збудження при віддаленні від джерела збудження, знайдено рішення хвильового рівняння коливань та виведені аналітичні вирази для визначення амплітудного напруження, яке виникає на поверхні та в основі ущільнюваного шару полімерного бетону залежно від координати. На підставі умови, яка описує ущільнюваність, отримано аналітичний вираз для визначення амплітуди збудження поверхні полімерного бетону, яка необхідна для його повного ущільнення. За визначеним значенням амплітуди збудження визначається амплітудне значення збуджуючої сили вібраторів для оснащення ними вібраційного робочого органу.

Ключові слова: реологічна модель, полімерний бетон, напруження, деформація, амплітуда збудження.

АКТУАЛЬНОСТЬ РАБОТЫ. Технологический процесс вибрационного уплотнения полимерного бетона реализуется путём взаимодействия вибрационного рабочего органа с полимерной бетонной смесью. При этом эффективность уплотнения полимерной бетонной смеси будет зависеть от технологических режимов вибрационного воздействия амплитуды и частоты колебаний вибрационного рабочего органа, а также продолжительности вибрационного воздействия.

В настоящее время при производстве изделий из полимерного бетона используется технология вибрационного уплотнения обычного цементного бетона или железобетона. Однако копирование технологических режимов и операций без должного учета специфических особенностей и свойств полимерной бетонной смеси не позволяет проявиться в должной мере положительным качествам конструкций из полимерного бетона. Кроме этого возможности теоретического анализа процесса вибрационного уплотнения полимерных бетонных смесей и точность получаемых результатов в значительной степени зависят от принятой расчетной реологической модели уплотняемой среды.

В проведенных ранее исследованиях поведение деформируемых сред моделировалось упругой моделью Гука, вязкого (модели Ньютона) или вязкоупругого (модели Кельвина - Фогта) тела, моделью Бингама [1-4], реологическими моделями, описывающими поведение сплошной среды с распреде- 
ленными параметрами [5-7]. Процесс релаксации напряжений в деформируемых средах в элементарном представлении описывался моделью Максвелла с последовательным сочетанием упругого и вязкого элементов, а также многоэлементными моделями Куна и Шкловского де Жена [8]. В некоторых случаях деформируемые среды представлялись математическими кривыми [9-12].

Наиболее точно уплотняемую полимерную бетонную среду можно представить реологической моделью Зинера [13], которая наряду с обратимой и необратимой деформацией описывает обратимую высокоэластичную деформацию, наиболее наглядно проявляющуюся у полимеров.

Однако в существующих исследованиях реологическая модель Зинера не применялась для описания поведения сплошной среды, а ее колебания, при действии вибрации, не описывались волновым уравнением колебаний.

Поэтому проведение теоретических исследований, направленных на научно-обоснованное определение амплитуды колебаний вибрационного рабочего органа и соответствующей ей возмущающей нагрузки, необходимых для уплотнения полимерного бетона, является достаточно актуальной задачей.

Цель работы - определение амплитуды колебаний и соответствующей ей вибрационной нагрузки, необходимых для уплотнения полимерных бетонных смесей.

МАТЕРИАЛ И РЕЗУЛЬТАТЫ ИССЛЕДОВАНИЙ. В настоящей работе для теоретического определения амплитуды колебаний и соответствующей вибрационной нагрузки, необходимых для уплотнения полимерных бетонных смесей, применен метод решения в комплексных функциях [12].

Для решения поставленной задачи полимерный бетон рассматривается как сплошная однородная среда, на которую действует возмущение $u(t)$ от вибрационного рабочего органа, которое с достаточной точностью может быть описано выражением [14]:

$$
u(0, t)=A \cdot \sin (\omega \cdot t)
$$

где $A$ - амплитуда смещения; $\omega$ - частота возмущения; $t$ - время.

Для выявления закона распространения волн напряжений полимерный бетон, находящийся под вибрационным рабочим органом, условно разделим на ряд элементарных объемов. Упруговязкопластические свойства полимерного бетона представим реологической моделью (рис. 1).

Для представленной реологической модели зависимость между напряжением и деформацией может быть описана уравнением:

$$
\begin{gathered}
\frac{\partial \sigma}{\partial t}+\sigma \cdot \frac{E_{2}}{\eta}=\left(E_{1}+E_{2}\right) \cdot \frac{\partial^{2} u(x, t)}{\partial x \partial t}+ \\
+\frac{E_{1} \cdot E_{2}}{\eta} \cdot \frac{\partial u(x, t)}{\partial x},
\end{gathered}
$$

где $u$ и $x$ - эйлерова и лагранжева координаты; $\sigma$ - напряжение, возникающее в рассматриваемом объёме полимерной бетонной смеси; $E_{1}$ и $E_{2}$ - модули упругой деформации полимерной бетонной смеси; $\eta$ - коэффициент динамической вязкости, учитывающий внутреннее трение в полимерной бетонной смеси.

Рассмотрим процесс деформирования полимербетонного слоя, схема которого приведена на рис. 1.
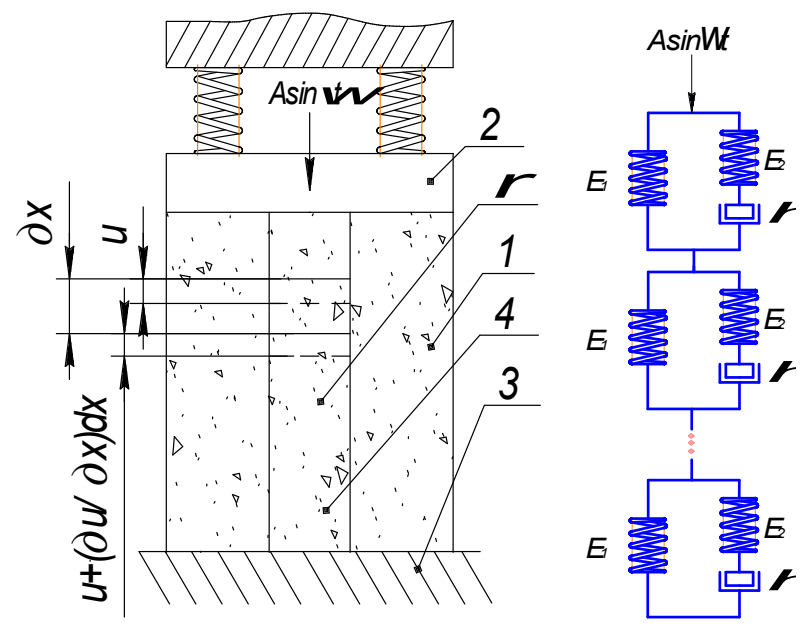

Рисунок 1 - Схема расчетной реологической модели уплотняемого полимерного бетона:

1 - полимерный бетон; 2 - вибрационный рабочий орган; 3 - основание слоя полимерного бетона;

4 - элементарный столб полимерного бетона из $n$ элементов

Будем считать, что плотность $\rho$ в элементарном объеме полимерного бетона постоянна и её изменения происходят скачкообразно при переходе от одного элементарного объёма к другому. Движение уплотняемой среды будем рассматривать только в направлении элементарного объёма и во времени.

Таким образом, для рассматриваемого одномерного случая дифференциальное уравнение движения среды будет иметь вид:

$$
\frac{\partial \sigma}{\partial x}=\rho \cdot \frac{\partial^{2} u}{\partial t^{2}},
$$

или с учетом (2):

$$
\begin{gathered}
\frac{\partial^{2} u(x, t)}{\partial x^{2}}+\eta \cdot\left(\frac{E_{1}+E_{2}}{E_{1} \cdot E_{2}}\right) \cdot \frac{\partial^{3} u(x, t)}{\partial x^{2} \partial t}-\left(\frac{\eta \cdot \rho}{E_{1} \cdot E_{2}}\right) \cdot \frac{\partial^{3} u(x, t)}{\partial t^{3}}- \\
-\left(\frac{\rho \cdot E_{2}}{E_{1} \cdot E_{2}}\right) \cdot \frac{\partial^{2} u(x, t)}{\partial t^{2}}=0
\end{gathered}
$$

Решение полученного волнового уравнения (4) будем отыскивать при следующих граничных условиях [14]:

$$
\begin{gathered}
u(0, t)=A \cdot \sin (\omega \cdot t) ; \\
u(H, t)=0,
\end{gathered}
$$


где $H$ - высота уплотняемого слоя полимерного бетона.

Решение уравнения (4) может быть представлено в виде мнимой части комплексного числа $[14,15]$ :

$$
u(x, t)=\operatorname{Im} \cdot\left[u(x) \cdot e^{i \omega \cdot t}\right],
$$

Знак при сомножителе Im в дальнейшем для удобства будем отбрасывать. Подставив зависимость (7) в выражение (4), получим уравнение для определения комплексной амплитуды колебаний:

$$
\begin{aligned}
& \left(1+i \eta \omega \cdot\left(\frac{E_{1}+E_{2}}{E_{1} \cdot E_{2}}\right)\right) \frac{\partial^{2} u(x)}{\partial x^{2}}+ \\
& +\frac{\rho \cdot \omega^{2}}{\mathrm{E}_{1}} \cdot\left(1+\frac{\mathrm{i} \eta \omega}{\mathrm{E}_{2}}\right) \cdot \mathrm{u}(\mathrm{x})=0 .
\end{aligned}
$$

Обозначим через $\widetilde{E}$ - комплексный модуль деформации:

$$
\widetilde{E}=1+i v_{1} \omega
$$

а через $\widetilde{\rho}$ - комплексную плотность полимерного бетона:

$$
\widetilde{\rho}=\rho_{1} \cdot\left(1+i v_{2} \omega\right)
$$

В зависимостях (9) и (10):

$$
v_{1}=\eta \cdot\left(\frac{E_{1}+E_{2}}{E_{1} \cdot E_{2}}\right) ; \quad \rho_{1}=\frac{\rho}{E_{1}} ; \quad v_{2}=\frac{\eta}{E_{2}} \text {. }
$$

При этом выражение (8) преобразуется к виду:

$$
\begin{aligned}
& \frac{\partial^{2} u(x)}{\partial x^{2}}+\frac{\widetilde{\rho} \cdot \omega^{2}}{\widetilde{E}} u(x)=0 . \quad(12) \\
& \alpha=\sqrt{\frac{\rho_{1} \cdot \omega^{2} \cdot\left[\sqrt{1+\omega^{2} \cdot\left(v_{1} \cdot v_{2} \cdot\left(2+\omega^{2} v_{1} \cdot v_{2}\right)+2 \cdot\left(v_{1}-v_{2}\right)^{2}\right)}-\left(1+\omega^{2} \cdot v_{1} \cdot v_{2}\right)\right]}{8 \cdot\left(1+\omega^{2} \cdot v_{1}^{2}\right)} ;} \\
& c=\sqrt{\frac{8 \cdot\left(1+\omega^{2} \cdot v_{1}^{2}\right)}{\rho_{1} \cdot\left[7 \cdot\left(1+\omega^{2} \cdot v_{1} \cdot v_{2}\right)+\sqrt{1+\omega^{2} \cdot\left(v_{1} \cdot v_{2} \cdot\left(2+\omega^{2} v_{1} \cdot v_{2}\right)+2 \cdot\left(v_{1}-v_{2}\right)^{2}\right)}\right]}} .
\end{aligned}
$$

Решение уравнения (12) будет иметь вид:

$$
u(x)=D_{1} \cdot e^{-i \tilde{k} x}+D_{2} \cdot e^{i \tilde{k} x}
$$

где $D_{1}$ и $D_{2}$ - постоянные интегрирования (комплексные амплитуды), определяемые граничными условиями (5) и (6); $\widetilde{k}$ - волновое число,

$$
\widetilde{k}=\omega \cdot \sqrt{\frac{\widetilde{\rho}}{\widetilde{E}}} .
$$

Если $\widetilde{E}$ и $\widetilde{\rho}-$ комплексные величины, то и волновое число также является комплексным. В этом случае его можно представить в виде комплексной функции [14-16]:

$$
\widetilde{k}=k-i \alpha=\frac{\omega}{c}-i \alpha,
$$

где $c$ - фазовая скорость распространения возмущения; $\alpha$ - коэффициент поглощения возмущения.

Для вычисления величин $c$ и $\alpha$ приравняем выражения (14) и (15), возведем левую и правую части в квадрат и, выделяя соответственно вещественную и мнимую части, получим систему уравнений:

$$
\left\{\begin{array}{l}
\frac{\rho_{1} \cdot \omega^{2} \cdot\left(1+\omega^{2} \cdot v_{1} \cdot v_{2}\right)}{1+\omega^{2} \cdot v_{1}^{2}}=\frac{\omega^{2}}{c^{2}}-\alpha^{2} ; \\
\frac{\rho_{1} \cdot \omega^{3} \cdot\left(v_{1}-v_{2}\right)}{1+\omega^{2} \cdot v_{1}^{2}}=\frac{2 \cdot \omega}{c} \cdot \alpha^{2}
\end{array}\right.
$$

откуда:
На основании зависимостей (13) и (15) выражение (7) преобразуется к следующему виду:

$$
u(x, t)=\left[D_{1} \cdot e^{-(\alpha+i k) \cdot x}+D_{2} \cdot e^{(\alpha+i k) \cdot x}\right] \cdot e^{i \omega \cdot t} .
$$

Подставляя (19) в граничное условие (6), найдем соотношение между постоянными интегрирования $D_{1}$ и $D_{2}$ в виде:

$$
D_{2}=-D_{1} \cdot \frac{e^{-(\alpha+i k) \cdot H}}{e^{(\alpha+i k) \cdot H}}
$$

Тогда выражение (19) преобразуется к виду:

$$
\begin{aligned}
u(x, t) & =D_{1} \cdot\left[\frac{e^{(\alpha+i k) \cdot(H-x)}-e^{-(\alpha+i k) \cdot(H-x)}}{e^{(\alpha+i k) \cdot H}}\right] \cdot e^{i \omega \cdot t}= \\
& =2 \cdot D_{1} \cdot\left[\frac{\operatorname{sh}[(\alpha+i k)(H-x)]}{e^{(\alpha+i k) \cdot H}}\right] \cdot e^{i \omega \cdot t} \cdot
\end{aligned}
$$

Путем подстановки выражения (21) в граничное условие (5), найдем постоянную интегрирования $D_{1}$ в следующем виде:

$$
D_{1}=\frac{A \cdot e^{(\alpha+i k) \cdot H}}{2 \cdot \operatorname{sh}[(\alpha+i k) \cdot H]} .
$$


Подставляя найденное значения постоянной интегрирования $D_{1}$ (22) в выражение (21), найдем решение уравнения (4), удовлетворяющее граничным условиям (5) и (6) в комплексной форме, т.е.

$$
u(x, t)=A \cdot\left[\frac{\operatorname{sh}[(\alpha+i k)(H-x)]}{\operatorname{sh}[(\alpha+i k) H]}\right] \cdot e^{i \omega \cdot t} \text {. }
$$

Преобразуем выражение (23), приведя его к следующему виду:

$$
\begin{aligned}
u(x, t)= & \frac{A}{\operatorname{sh}(\alpha H) \cdot \cos (k H)+i \cdot \operatorname{ch}(\alpha H) \cdot \sin (k H)} \times \\
& \times\{\operatorname{sh}[\alpha(H-x)] \cdot \cos [k(H-x)]+ \\
& +i \cdot \operatorname{ch}[\alpha(H-x)] \cdot \sin [k(H-x)]\} \cdot e^{i \omega t} . \quad(24)
\end{aligned}
$$

Умножая, числитель и знаменатель зависимости (24) на комплексную функцию, сопряженную знаменателю, и выделяя из полученного выражения мнимую часть, получим искомое решение уравнения (4), удовлетворяющее граничным условиям (5) и (6) в следующем виде:

$$
\begin{aligned}
& u(x, t)=\frac{A}{\sqrt{\operatorname{sh}^{2}(\alpha H) \cos ^{2}(k H)+\operatorname{ch}^{2}(\alpha H) \sin ^{2}(k H)}} \times \\
& \times\{\operatorname{sh}[\alpha(H-x)] \cdot \cos [k(H-x)] \cdot \sin (\omega t-\varphi)+ \\
& +\operatorname{ch}[\alpha(H-x)] \cdot \sin [k(H-x)] \cdot \cos (\omega t-\varphi)\},(25)
\end{aligned}
$$

где $\varphi$ - угол сдвига фаз,

$$
\begin{aligned}
\varphi & =\operatorname{arctg}\left[\frac{\operatorname{ch}(\alpha H) \cdot \sin (k H)}{\operatorname{sh}(\alpha H) \cdot \cos (k H)}\right]= \\
& =\operatorname{arctg}[\operatorname{cth}(\alpha H) \cdot \operatorname{tg}(k H)] .
\end{aligned}
$$

Подставляя решение (26) в уравнение (3), определим напряжение, возникающее в уплотняемом слое в зависимости от координаты $x$ :

$$
\begin{aligned}
& \sigma(x, t)=- \frac{A \cdot \rho \cdot \omega^{2}}{\left(\alpha^{2}+k^{2}\right) \sqrt{\operatorname{sh}^{2}(\alpha H) \cos ^{2}(k H)+\operatorname{ch}^{2}(\alpha H) \sin ^{2}(k H)}} \cdot \\
& \cdot[\{k \cdot \operatorname{sh}[\alpha(H-x)] \sin [k(H-x)]+ \\
&+\alpha \cdot \operatorname{ch}[\alpha(H-x)] \cos [k(H-x)]\} \cdot \sin (\omega t-\varphi)- \\
&-\{\alpha \cdot \operatorname{sh}[\alpha(H-x)] \sin [k(H-x)]+ \\
&+k \cdot \operatorname{ch}[\alpha(H-x)] \cos [k(H-x)]\} \cdot \cos (\omega t-\varphi)] \cdot(27)
\end{aligned}
$$

Подставляя в выражение (27) значения координаты $x=0$ и $x=H$, определим изменение напряжений, возникающих на поверхности

$$
\begin{aligned}
& \sigma(0, t)=-\frac{A \cdot \rho \cdot \omega^{2}}{\left(\alpha^{2}+k^{2}\right) \sqrt{\operatorname{sh}^{2}(\alpha H) \cos ^{2}(k H)+c h^{2}(\alpha H) \sin ^{2}(k H)}} . \\
& \cdot[\{k s h(\alpha H) \sin (k H)+\alpha \operatorname{ch}(\alpha H) \cos (k H)\} \sin (\omega t-\varphi)-
\end{aligned}
$$

$$
\begin{gathered}
-\{\alpha \cdot \operatorname{sh}(\alpha H) \sin (k H)+k \cdot \operatorname{ch}(\alpha H) \cos (k H)\} \times \\
\times \cos (\omega t-\varphi)],
\end{gathered}
$$

и в основании полимерного слоя бетона:

$$
\begin{gathered}
\sigma(H, t)=-\frac{A \cdot \rho \cdot \omega^{2} \cdot \sqrt{\alpha^{2}+k^{2}}}{\left(\alpha^{2}+k^{2}\right)} \times \\
\times \frac{\sin (\omega t-\varphi-\psi)}{\sqrt{\operatorname{sh}^{2}(\alpha H) \cos ^{2}(k H)+\operatorname{ch}^{2}(\alpha H) \sin ^{2}(k H)}}, \\
\text { где } \quad \psi=\operatorname{arctg}\left[\frac{k}{\alpha}\right]
\end{gathered}
$$

Из выражения (27) следует, что напряжение, возникающие в уплотняемом слое, зависит от толщины слоя $H$, коэффициента поглощения возмущения $\alpha$, волнового числа $k$, плотности полимерной бетонной смеси $\rho$, амплитуды $A$ и частоты возмущения $\omega$.

Амплитудное напряжение прямо пропорционально амплитуде возмущения $A$ и для того, чтобы получить требуемую плотность полимерного бетона по всей толщине уплотняемого слоя, необходимо выбрать значение величины $A$ из условия уплотняемости $[12,14]$ :

$$
\sigma(H, t) \geq \sigma_{0 i}
$$

где $\sigma_{0 i}$ - минимальное напряжение, обеспечивающее предельное разрушение структуры полимербетонной смеси и наиболее эффективное уплотнение.

Тогда из условия уплотняемости (32):

$$
\begin{gathered}
A=\frac{\sigma_{0 i} \cdot\left(\alpha^{2}+k^{2}\right)}{\rho \cdot \omega^{2} \cdot \sqrt{\alpha^{2}+k^{2}}} \times \\
\times \frac{\sqrt{\operatorname{sh}^{2}(\alpha H) \cos ^{2}(k H)+c h^{2}(\alpha H) \sin ^{2}(k H)}}{\sin (\omega t-\varphi-\psi)} .
\end{gathered}
$$

На основании амплитуды возмущения поверхности полимерного бетона $A$, требуемой для его полного уплотнения, определяется амплитудное значение возмущающей силы вибраторов вибрационного рабочего органа $Q$.

ВЫВОДЫ. В результате проведенных теоретических исследований определена амплитуда колебаний вибрационного рабочего органа и соответствующая ей вибрационная нагрузка, необходимая для уплотнения полимерных бетонных смесей. На основании найденного значения амплитуды возмущения поверхности полимерного бетона, требуемой для его полного уплотнения, определяется амплитудное значение возмущающей силы вибраторов вибрационного рабочего органа. 


\section{ЛИТЕРАТУРА}

1. Chen X., Wu S., Zhou J. Experimental study and analytical formulation of mechanical behavior of concrete. Construction and Buildings Materials. 2013. Vol. 47. P. 662-670.

2. Tattersall G. H. Effect of Vibration on the Rheological Properties of Fresh Cement Pastes and Concretes. Rheology of Fresh Cement and Concrete, Proceedings of the International Conference, P. F. G. Banfill, ed., University of Liverpool, UK, Mar. 16-29, Chapman and Hall, London. 1990. P. 323-338.

3. Kakuta S., Kojima T. Rheology of Fresh Concrete under Vibration. Rheology of Fresh Cement and Concrete, Proceedings of the International Conference, P. F. G. Banfill, ed., University of Liverpool, UK, Mar. 16-29, Chapman and Hall, London. 1990. P. 339-342.

4. P. F. G. Banfill, et al. Rheology and vibration of fresh concrete: Predicting the radius of action of poker vibrators from wave propagation. Cement and Concert Research. 2011. Vol. 41, № 9. P. 932-941.

5. Маслов А. Г. Иткин А. Ф., Саленко Ю. С. Вибрационные машины для приготовления и уплотнения бетонных смесей: Кременчуг: ЧП Щербатых А. В, 2014. 324 с.

6. Маслов А. Г., Жанар Батсайхан. Исследование колебаний рабочего органа машины для уплотнения бетонных смесей в вибрационном рабочем режиме. Вісник Кременчуцького національного університету імені Михайла Остроградського, 2015. Вип. 2 (91), частина 1. С. 92-97.

7. Маслов А. Г., Иткин А. Ф. Теоретические основы вибрационного уплотнения цементобетонных смесей. Вісник Кременчуиьького державного політехнічного університету. 2004. Вип. 5. С. 45-49.

8. Бобрышев А. Н., Ерофеев В. Т., Козомахов В. Н. Физика и синергетика дисперсно-неупорядоченных конденсированных композитных систем. Наука, Санкт-Петербург, 2012. 473 с.

9. $\mathrm{Hu}$ C., Larrard F. The Rheology of Fresh HighPerformance Concrete. Cement and Concrete Research. 1996. Vol. 26, №. 2. P. 283-294.

10. Szwabowski J. Influence of Three-Phase Structure on the Yield Stress of Fresh Concrete. Rheology of Fresh Cement and Concrete, Proceedings of the International Conference, P. F. G. Banfill, ed., University of Liverpool, UK, Mar. 16-29, 1990, Chapman and Hall, London. 1990. P. 241-248.

11. Kłosiński J., Trąbka A. Frequency analysis of vibratory device model (in Polish). Pneumatyka. 2010. Vol. 1. P. 46-49.

12. Żółtowski B. Research of machine dynamics (in Polish). Wyd. MARKAR, Bydgoszcz. 2002.

13. Бобрышев А. Н., Воронов П. В., Галимов Э. Р., Лахно А. В., Абдуллин И. А. Кинетические модели релаксации напряжений в композитах. Вестник Технологического университета. 2014. Вып. 17/2014 (14). С. 431-434.

14. Холодов А. М., Маслов А. Г. Определение возмущающей нагрузки, необходимой для уплотнения асфальтового бетона. Строительные и дорожные машины. 1973. Вип. 16. С. 58-64.

15. Маслов А. Г., Иткин А. Ф. Исследование процесса уплотнения цементной бетонной смеси на вибрационной площадке с вертикально направленными колебаниями. Вісник Кременчуцького наиіонального університету імені Михайла Остроградського. 2004. Вип. 6/2004 (29). С. 50-54.

16. Маслов А. Г., Пономарь В. М. Вибрационные машины и процессы в дорожном строительстве. Київ : Будівельник, 1985. 128 с.

\section{A. Maslov, D. Savielov}

Kremenchuk Mykhailo Ostrohradskyi National University

vul. Pershotravneva, 20, Kremenchuk, 39600, Ukraine. E-mail: dvsavelov@gmail.com

Purpose. On the basis of a thorough analysis of scientific and technical literature, it has been established that to date, for the production of products from polymer concrete, the technology of vibration sealing of cement concrete or reinforced concrete is used, which does not take into account the specific features and properties of the polymer concrete mix and does not allow to receive qualitative and reliable products from it. Methodology. On the basis of the analysis of the behavior of the materials and the existing rheological models of sealing media, it was determined that for the vibrating polymeric concrete mixture a Ziener rheological model was proposed in which, under the action of the external load together with the reversible and irreversible deformation, the reversible high elastic deformation of the polymer binder is taken into account. Results. It was established that in the existing research, the Ziener rheological model was not used to describe the behavior of a polymeric concrete mixture as a continuous medium, and its oscillations, under the influence of vibration, were not described by the wave equation of oscillation. For the proposed rheological model of the sealing polymer concrete mixture, which takes into account its elastic, viscous and plastic properties, a wave equation of oscillations was made, for the theoretical analysis of which the method of solution in complex functions is used. As a result of the theoretical studies, the phase rate of the excitation propagation and the absorption coefficient were determined, which characterizes the decrease in excitation at the distance from the source of excitation, a solution of the wave equation of oscillation was found, and the analytical expressions were derived for determining the amplitude stress that occurs on the surface and on the basis of the sealing layer of polymeric concrete depending from the coordinate. Originality. Based on the condition that describes the seal, an analytical expression was obtained to determine the amplitude of the excitation of the surface of the polymeric concrete, which is necessary for its complete sealing. Practical value. By the determined value of the amplitude of the excitation, the amplitude value of the excitatory force of the vibrators of the vibrational working body is determined.

Key words: rheological model, polymer concrete, stress, deformation, amplitude of excitation. 


\section{REFERENCES}

1. Chen, X., Wu, S., Zhou, J. (2013), Experimental study and analytical formulation of mechanical behavior of concrete, Construction and Buildings Materials 47, pp. 662-670.

2. Tattersall, G. H. (1990), Effect of Vibration on the Rheological Properties of Fresh Cement Pastes and Concretes, Rheology of Fresh Cement and Concrete, Proceedings of the International Conference, $P . F$. G. Banfill, ed., University of Liverpool, UK, Mar. 16-29, Chapman and Hall, London, pp. 323-338.

3. Kakuta, S., Kojima, T. (1990), "Rheology of Fresh Concrete under Vibration, Rheology of Fresh Cement and Concrete, Proceedings of the International Conference, P. F. G. Banfill, ed., University of Liverpool, UK, Mar. 16-29, Chapman and Hall, London, pp. 339-342.

4. Banfill, P. F. G et al. (2011), "Rheology and vibration of fresh concrete: Predicting the radius of action of poker vibrators from wave propagation," Cement and Concert Research, vol. 41, no. 9, pp. 932-941.

5. Maslov, A. G., Itkin, A. F., Salenko, Y. S. (2014), Vibratsionnyie mashinyi dlya prigotovleniya $i$ uplotneniya betonnyih smesey [Vibrating machines for the preparation and compaction of concrete mixes] PP Cherbatyh, Kremenchuk, Ukraine.

6. Maslov, A. G., Batsaikhan, Zhanar (2015), "The Research of oscillations of the machine working body of the for compaction of concrete mixes in vibration working mode", Transactions of Kremenchuk Mykhailo Ostrohradskyi National University. Kremenchug: KRNU, Issue (91), pp. $92-97$.

7. Maslov, A. G., Itkin, A. F. (2004), "Theoretical foundations of the vibration compaction of concrete mix", Transactions of Kremenchuk Mykhailo Ostrohradskyi State Polytechnic University. Kremenchug: KDPU, Issue (28), pp. 45 - 49.

8. Bobryshev, A. N., Erofeev, V. T., Kozomahov, V. N. (2012), Fizika i sinergetika dispersnoneu- poryadochennyh kondensirovannyh kompozitnyh sistem [Physics and synergetics of dispersed disordered condensed composite systems], Nauka, SanktPeterburg, Russia.

9. Hu, C., Larrard, F. (1996), "The Rheology of Fresh High-Performance Concrete", Cement and Concrete Research, Vol. 26, No. 2, pp. 283-294.

10. Szwabowski, J. (1990), Influence of Three-Phase Structure on the Yield Stress of Fresh Concrete, Rheology of Fresh Cement and Concrete, Proceedings of the International Conference, P. F. G. Banfill, ed., University of Liverpool, UK, Mar. 16-29, 1990, Chapman and Hall, London, pp. 241-248.

11. Kłosiński, J., Trąbka, A. (2010), Frequency analysis of vibratory device model (in Polish), Pneumatyka, Vol. 1, pp. 46-49.

12. Żółtowski, B. (2002), Research of machine dynamics (in Polish), Wyd. MARKAR, Bydgoszcz.

13. Bobryshev, A. N., Voronov, P. V., Galimov, E. R., Lahno, A. V., Abdullin, I. A. (2014), "The kinetic models of stress relaxation in composites", Transactions of technological University, Issue 17/2014 (14), pp. 431 434.

14. Holodov, A. M., Maslov, A. G. (1973), "Determination of perturbing forces required for compaction of asphalt concrete", Build and travelling machines, Issue 16, pp. $58-64$.

15. Maslov, A. G., Itkin, A. F. (2004), "Research of process of compression of cement concrete mixture on an oscillation ground with the apeak directed vibrations", Transactions of Kremenchuk Mykhailo Ostrohradskyi State Polytechnic University. Kremenchug: KDPU, Issue (29), pp. 50 - 54.

16. Maslov, A. G., Ponomar', V. M. (1985), Vibracionnye mashiny i processy $\mathrm{v}$ dorozhnom stroitel'stve [Vibration machines and processes are in travelling building], Budivel'nik, Kiev, Ukraine.

Стаття надійшла 18.03.2019. 